\title{
Blob motion and control in simple magnetized plasmas ${ }^{a)}$
}

\author{
C. Theiler, ${ }^{\text {b) }}$ I. Furno, A. Fasoli, P. Ricci, B. Labit, and D. Iraji \\ Centre de Recherches en Physique des Plasmas-Ecole Polytechnique Fédérale de Lausanne (EPFL), \\ Association EURATOM-Confédération Suisse, CH-1015 Lausanne, Switzerland
}

(Received 18 November 2010; accepted 19 January 2011; published online 2 May 2011)

\begin{abstract}
The radial propagation of plasma blobs and possibilities of influencing it are investigated in the TORPEX toroidal experiment [Fasoli et al., Phys. Plasmas 13, 055902 (2006)]. The effect of changing the connection length and the neutral background pressure on blob velocity is measured and trends are found to agree with predictions from a previous study [Theiler et al., Phys. Rev. Lett. 103, 065001, (2009)]. Effects on blob motion due to a change in limiter material and geometry are also discussed. (C) 2011 American Institute of Physics. [doi:10.1063/1.3562944]
\end{abstract}

\section{INTRODUCTION}

Plasma particles and energy can efficiently be convected across a confining magnetic field in the form of blobs, coherent structures of enhanced plasma density relative to the background plasma. Blobs are filaments, as they typically extend much further along the magnetic field than perpendicularly to it. They are measured near the edge of magnetized laboratory plasmas such as tokamaks, stellarators, reversed field pinches, simple magnetized tori, and linear devices. ${ }^{1-9}$

Besides being of fundamental physics interest, the dynamics of these structures in fusion devices influence location and strength of heat and particle fluxes to the divertor or first wall, impurity screening characteristics, wall recycling, and possibly the global confinement properties. ${ }^{10-12}$

Blob propagation can qualitatively be understood as follows: ${ }^{13}$ charge dependent drifts, such as those generated by magnetic field gradients and curvature, lead to cross-field currents and, due to the blob spatial inhomogeneity across the magnetic field, to charge separation. The resulting electric field inside the blob gives rise to an $\mathbf{E} \times \mathbf{B}$ motion. The magnitude of the cross-field velocity depends on the available current paths to damp charge separation. A large theoretical effort has been undertaken over the past few years to elucidate the dynamics of blob propagation in different regimes and geometries (see Ref. 14 and references therein). The experimental verification of such predictions is an active area of research. ${ }^{15-22}$

Recently, we have studied plasma blob motion in the TORPEX (Refs. 23 and 24) toroidal device, characterized by a simple open magnetic field line geometry. In the investigated scenario, blobs form from an interchange wave ${ }^{7}$ and propagate radially outwards. We have interpreted their motion using a $2 \mathrm{D}$, sheath limited blob model, ${ }^{13,25}$ in which parallel currents are determined by sheath boundary conditions. Theoretical studies of this model show that coherent blob motion is limited to $\tilde{a} \sim 1$, where $\tilde{a}$ is a normalized blob vertical size. ${ }^{26,27}$ For $\tilde{a} \gtrsim 1$, sheath currents dominate over inertia and blob radial velocity scales as $1 / \tilde{a}^{2}$. This is analytically derived in the original paper ${ }^{13}$ and observed in

\footnotetext{
a) Paper GI3 4, Bull. Am. Phys. Soc. 55, 109 (2010).

${ }^{\mathrm{b})}$ Invited speaker.
}

simulations, e.g., in Ref. 28. For $\tilde{a} \lesssim 1$, inertia, the divergence of ion-polarization currents, is the dominant term that limits blob propagation. In this limit, a blob initially at rest accelerates and reaches an approximately constant radial velocity $\propto \sqrt{\tilde{a}} .^{29,30}$ By using different gases, we have varied for the first time in an experiment the normalized blob size $\tilde{a}$ between the two regimes, $\tilde{a} \gtrsim 1$ and $\tilde{a} \lesssim 1 .^{21}$ Considering also cross-field ion currents due to a neutral friction force, we have derived an analytical expression for blob velocity in the case of interchange dominated turbulence. This expression retrieves the dependencies $1 / \tilde{a}^{2}$ and $\sqrt{\tilde{a}}$ in the appropriate limits and agrees well with our experimental measurements.

The purpose of the present paper is to consolidate the previous interpretation of blob motion and explore the insights gained from our previous study to actively influence blob propagation in TORPEX. In Sec. II, we discuss the blob velocity scaling law. In Secs. III and IV, we review the experimental setup and previous results, and describe the analysis techniques adopted in this work. We then investigate the possibility of influencing blob motion by varying the connection length and the neutral gas pressure in Secs. V and VI. In Sec. VII, we discuss experiments in which blob control is attempted by changing the blob boundary conditions. Section VIII summarizes and discusses further steps.

\section{BLOB VELOCITY SCALING}

In this section, we discuss the blob velocity scaling derived in Ref. 21. We consider a geometry with constant curvature along the field lines, constant connection length, and perpendicular incidence of the magnetic field lines on a conducting wall. In TORPEX, such a setup is achieved by inserting a steel limiter into the vacuum vessel, as indicated in Fig. 1(a). Assuming a constant electron temperature, cold ions $\left(T_{i} \ll T_{e}\right.$ ) for the electron heated TORPEX plasmas, ${ }^{31}$ and a $2 \mathrm{D}$ structure of the blob, the condition for quasineutrality $(\nabla \cdot \mathbf{J}=0)$ takes the following form: ${ }^{14}$

$$
\frac{2 c_{s}^{2} m_{i}}{R B} \frac{\partial n}{\partial z}=\frac{m_{i}}{B^{2}} \nabla \cdot\left(n \frac{D \nabla_{\perp} \phi}{D t}+n v_{i n} \nabla_{\perp} \phi\right)-\frac{n e^{2} c_{s}}{T_{e} L} \tilde{\phi} .
$$

Here, $c_{s} \approx \sqrt{T_{e} / m_{i}}$ is the ion sound speed, $m_{i}$ is the ion mass, $n$ is the plasma density, $B$ is the magnetic field 

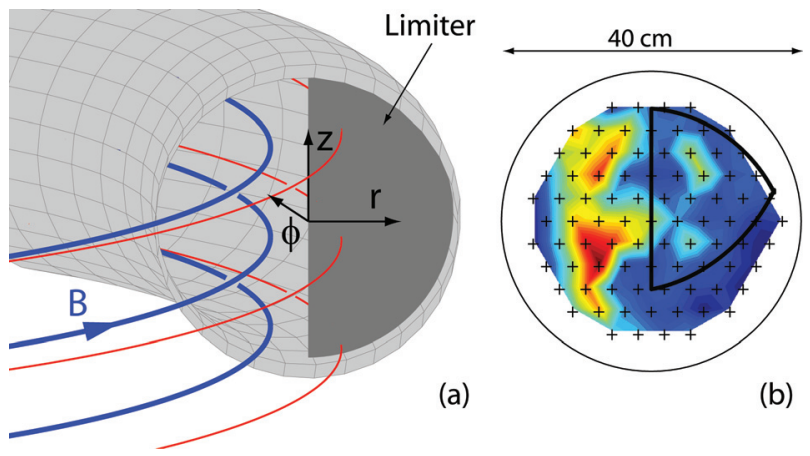

(a)

(b)

FIG. 1. (Color online) (a) Sketch of the experimental setup with two examples of helical magnetic field lines. The thick blue line lies in the main plasma region and does approximately three turns before intercepting the vacuum vessel. The thin red line lies in the blob region and intercepts the limiter after one turn. (b) Instantaneous $I_{\text {sat }}$ profile in the plane perpendicular to the magnetic field with two blob examples on the right-hand side. The arrangement of Langmuir probe tips (crosses) and the area where both field line ends are connected to the limiter (black contour) are also indicated.

(oriented as in Fig. 1), $L$ is the connection length, $R$ is the major radius, $\phi$ is the electrostatic plasma potential, and $\tilde{\phi}=\phi-\phi_{f}$ is the deviation from its floating value $\phi_{f} \approx$ $3 T_{e} /$ e. $\quad v_{\text {in }}$ is the ion-neutral collision frequency and $D / D t=\partial / \partial t+\mathbf{v}_{\mathbf{E} \times \mathbf{B}} \cdot \nabla$. The term on the left-hand side is the divergence of the electron diamagnetic current, the drive for blob motion. The current loop is closed by ion-polarization currents, ion currents caused by a neutral friction force, and sheath currents. These effects are taken into account by the three terms on the right-hand side of Eq. (1). The sheath current term differs by a factor of two compared to some simulations. This is because we assume a density at the sheath edge, which is half the upstream density.

We now estimate the magnitude of the different terms in Eq. (1). We assume that the density blob is a monopole structure and the potential blob is a dipole with a positive and negative pole at the top and at the bottom of the blob, respectively. This agrees with our measurements in Fig. 3. We define $a$ as the half width at half maximum of the vertical cut of the density profile of the blob and estimate the terms of Eq. (1) at the position of the positive pole of the potential as follows: $\partial n / \partial z \sim-\delta n / a, \nabla_{\perp} \phi=0, \nabla^{2} \phi \sim-\tilde{\phi} / a^{2}$, and $\tilde{\phi} \sim B v_{\text {blob }} a$. Further, we assume that the blob is subject to secondary instabilities with a growth rate $\gamma_{\text {inst }}$ that can limit its motion. ${ }^{32}$ Setting $D / D t \sim \gamma_{\text {inst }}$, we obtain for the blob radial velocity $v_{\text {blob }}$

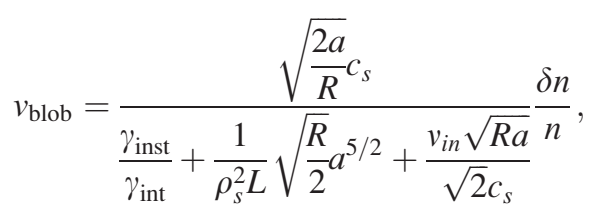

with $\gamma_{\text {int }}=\sqrt{2} c_{s} / \sqrt{R a}$ the ideal interchange growth rate and $\rho_{s} \approx \sqrt{T_{e} m_{i}} /(e B)$ the ion sound Larmor radius. The terms in the denominator represent the damping of blob velocity due to inertia, parallel currents to the sheath, and ion-neutral collisions, respectively. The factor $\delta n / n$ (blob density above the background level divided by total blob density) describes the slowing down by a finite background density. ${ }^{16}$
As in Ref. 21 , we assume $\gamma_{\text {inst }} \approx \gamma_{\text {int }}$. In simulations, small blobs are found to be subject to the Kelvin-Helmholtz instability. ${ }^{14,26-28}$ In Appendix B, we discuss therefore the choice $\gamma_{\text {inst }} \approx v_{\text {blob }} / a$ and find a very similar result as with $\gamma_{\text {inst }} \approx \gamma_{\text {int }}$.

In the limit where sheath losses and ion-neutral collisions are negligible, we obtain $v_{\text {blob }}=\sqrt{2 a / R} c_{s}$, similar to Refs. 29 and 30. This limit is further motivated in Appendix A. If sheath losses become dominant, we find the scaling $v_{\text {blob }}=2(L / R)\left(\rho_{s}^{2} / a^{2}\right) c_{s}$ of Ref. 13; when ion-neutral friction dominates, we retrieve $v_{\text {blob }}=2 c_{s}^{2} /\left(R v_{i n}\right)$, which is derived and experimentally verified in Ref. 20. Further, for $\delta n / n \rightarrow 1$ and $v_{i n} \rightarrow 0$ in Eq. (2), we find good agreement with simulation results in Ref. 28 .

Using dimensionless quantities, $\tilde{a}=a / a^{*}$ and $\tilde{v}_{\text {blob }}$ $=v_{\text {blob }} / v_{\text {blob }}^{*}$, where $a^{*}$ and $v_{\text {blob }}^{*}$, similarly to Ref. 14 , and references therein, are defined as

$$
a^{*}=\left(\frac{4 L^{2}}{\rho_{s} R}\right)^{1 / 5} \rho_{s}, \quad v_{\mathrm{blob}}^{*}=\left(\frac{2 L \rho_{s}^{2}}{R^{3}}\right)^{1 / 5} c_{s},
$$

we obtain the following form of Eq. (2):

$$
\tilde{v}_{\text {blob }}=\frac{\sqrt{2 \tilde{a}} \delta n / n}{1+\sqrt{2} \tilde{a}^{5 / 2}+\tilde{\eta} \sqrt{\tilde{a}}}, \quad \tilde{\eta}=\frac{v_{\text {in }} \rho_{s}}{c_{s}}\left(\frac{L R^{2}}{\sqrt{8} \rho_{s}^{3}}\right)^{1 / 5} .
$$

This expression shows the importance of the different damping mechanisms, which depend upon $\tilde{a}$ and, through $\tilde{\eta}$, upon the ion-neutral collision frequency. The three limits discussed above correspond thus to $\tilde{a}^{5 / 2}, \tilde{\eta} \sqrt{\tilde{a}} \ll 1$, to $\tilde{a}^{5 / 2} \gg 1, \tilde{\eta} \sqrt{\tilde{a}}$, and to $\tilde{\eta} \gg 1 / \sqrt{\tilde{a}}, \tilde{a}^{2}$.

\section{EXPERIMENTAL SETUP AND PREVIOUS RESULTS}

Experiments are performed in the simple magnetized toroidal device TORPEX (Refs. 23 and 24) (major radius $R=1 \mathrm{~m}$ ), where plasmas are produced and sustained by microwaves in the electron cyclotron range of frequencies. ${ }^{31}$

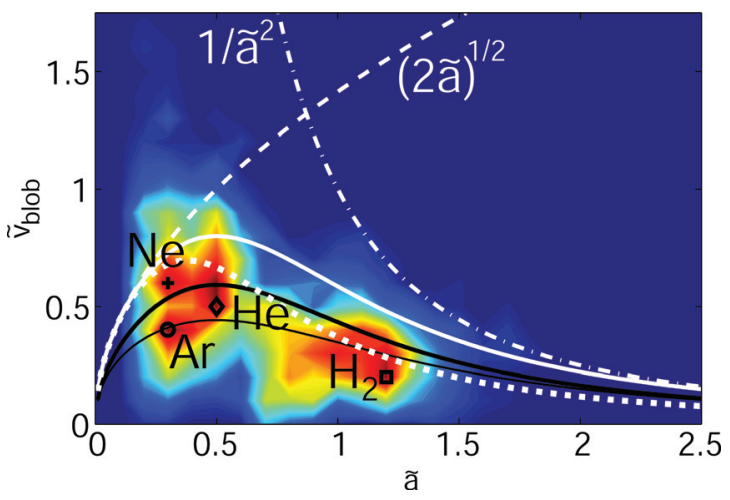

FIG. 2. (Color online) Joint probability of normalized blob radial velocity versus blob size from Ref. 21. Superimposed are the scaling laws $\tilde{v}_{\text {blob }}=\sqrt{2 \tilde{a}}$ and $\tilde{v}_{\text {blob }}=1 / \tilde{a}^{2}$ (dashed and dash-dotted white lines), as well as the scaling law in Eq. (4) (solid white) with corrections due to a background of plasma (thick black) and additionally a background of neutrals (thin black). The symbols indicate the peaks of the distributions for different ion masses (working gases): hydrogen (square), helium (diamond), neon (plus), and argon (circle). The white dotted curve shows the change in blob velocity with respect to the solid, white curve, following a doubling of the sheath damping. 
The magnetic field consists of a dominant toroidal field component of $\approx 0.08 \mathrm{mT}$ on which a small vertical magnetic field $B_{z}$ is superimposed. This results in helical field lines that intercept the vacuum vessel at the bottom and the top, as sketched in Fig. 1. The nature of the dominant instability can be controlled by the strength of $B_{z} \cdot{ }^{33,34}$ In this work, we focus on the ideal interchange regime that has already been extensively investigated in TORPEX. A relatively low injected microwave power results in vertically elongated, slablike profiles that peak on the high-field side, i.e., on the inner side of the device cross section. An ideal interchange wave develops in the low-field side region, where pressure gradients and magnetic field gradients are collinear. Blobs are generated from the ideal interchange wave and propagate radially outwards, ${ }^{7}$ exhibiting universal statistical properties similar to the scrapeoff layer (SOL) of fusion plasmas ${ }^{35}$ and contributing significantly to cross-field particle transport. ${ }^{36,37}$ Studies of blob generation in this setup have revealed that blobs form from radially extending positive crests of the wave that get sheared apart by the $\mathbf{E} \times \mathbf{B}$ flow. ${ }^{7,37}$ The radial elongation of the wave is attributed to a steepening of the pressure profile. ${ }^{7,38}$

In order to experimentally reproduce the situation modeled by Eq. (1) in Ref. 21, we have inserted a steel limiter in the blob region, i.e., the region where the dynamics is characterized by radially propagating blobs (see Fig. 1). From Eq. (3), we see that $a^{*} \alpha \rho_{s}^{4 / 5} \alpha m_{i}^{2 / 5}$. In the experiments, the ion mass can be strongly varied by using different gases. Therefore, to change the normalized blob size $\tilde{a}$ and to access both the regime dominated by parallel currents and by inertia, respectively, we have used four different gases $\left(\mathrm{H}_{2}\right.$, $\mathrm{He}, \mathrm{Ne}$, and Ar) in Ref. 21. The result is displayed in Fig. 2, which shows the joint probability of normalized vertical blob size versus normalized radial velocity obtained using pattern recognition. ${ }^{21,39}$ Since $\tilde{a} \propto a\left(T_{e} m_{i}\right)^{-2 / 5}$, and $a$ and $T_{e}$ do not vary strongly from gas to gas, $\tilde{a}$ decreases with the ion mass and approaches the regime where parallel currents are unimportant. The white solid curve in Fig. 2 represents the scaling law Eq. (4) for $\delta n / n=1$ and $v_{i n}=0$. Including a finite background plasma and, additionally, the presence of neutrals results in the thick and thin black curves. The white dotted curve differs from the white one by the assumption of a twice as high sheath dissipation term. It illustrates the expected effect of halving the connection length on blob velocity in the different regimes (we assume here that a variation in connection length enters in Eq. (4) and not in the definition of $a^{*}$ and $v$ tob. In reality, a variation of $L$ changes $\tilde{a}$ and $\tilde{v}_{\text {blob }}$ and the expected change in blob velocity is harder to access in normalized units). The relative difference between the white dotted and the white curve increases as we increase $\tilde{a}$, thus accessing the regime where parallel currents become more and more important.

We have, therefore, two predictions. First, reducing the connection length should influence blob motion for $\mathrm{He}$ and more importantly for $\mathrm{H}_{2}$ blobs in TORPEX. Second, increasing the background gas pressure should reduce blob velocity. In Secs. V and VI, we will experimentally test these two predictions.

We will use the same target plasmas as in Ref. 21, except for discharges in Ar. Argon ionizes very easily and it is difficult to produce profiles that are limited to the high field side with a level of $\approx 300 \mathrm{~W}$ of injected microwave power. Therefore, we use a low power source ${ }^{40}$ to create the desired argon plasmas. As an example of the target plasmas, we show in Fig. 1 a snapshot of ion saturation current measured across the poloidal plane with the 2D Langmuir probe array HEXTIP (Ref. 41) in a He plasma. Here, the time-averaged profile peaks on the high-field side. On the low-field side, two blobs can be identified. The region where field lines terminate with both ends on a limiter, in both cases, for one and for two limiters, is indicated by the black contour. We will focus on blobs in this region.

\section{BLOB ANALYSIS}

To analyze blob motion, we use two different techniques. One is a pattern recognition technique applied to data from the HEXTIP probe array. ${ }^{39}$ The other one is a modification of the standard conditional sampling technique, ${ }^{42}$ which allows measuring the average 2D evolution of $n, T_{e}$, and $V_{f l}$ associated with a blob.

The pattern recognition method defines structures as regions where ion saturation current fluctuations $\delta I_{\text {sat }}(r, z, t)$ : $=I_{\mathrm{sat}}(r, z, t)-\left\langle I_{\mathrm{sat}}(r, z)\right\rangle_{t}$ exceed a threshold value. It then tracks these structures frame by frame and determines structure-related quantities such as the radial velocity. From this, we can evaluate radial profiles of the average radial velocity of the detected structures. We select all structures that exist for at least ten time frames, corresponding to $36 \mu \mathrm{s}$ (the double for the relatively slow blobs in argon) and that do not undergo merging or splitting events ${ }^{39}$ during that time. We will use a fixed threshold value for the analysis in a given working gas $\left(\approx 0.75 \sigma_{\mathrm{tot}}\right.$, where $\sigma_{\mathrm{tot}}^{2}=\sum_{i} \sigma_{i}^{2}$ and the sum is performed over all HEXTIP tips and $\sigma_{i}$ is the standard deviation of the $i$ th $\operatorname{tip}^{39}$ ). We have verified that our results depend weakly on the chosen threshold value. With this analysis, we detect all turbulent structures, including those that do not propagate or even move slightly inwards and that we do not expect to be described by the scaling law Eq. (2). In Ref. 21, we have used additional selection criteria to detect "wellbehaving" blobs. These are not used in the present analysis.

Since the pattern recognition technique does not allow measuring the blob temperature and its variation as the second limiter is installed, we use a modified conditional sampling method to further quantify our results. This method, described in detail in Ref. 43 and benchmarked with a triple probe in Ref. 44, can be illustrated as follows. A fixed reference probe in the blob region measures ion saturation current, while one or several movable probes are operated in swept mode. Here, we will use a vertically oriented eight-tip linear Langmuir probe array, referred to as SLP, as the movable probe. In the $I_{\text {sat }}$ time trace of the reference probe, we are detecting positive bursts, interpreted to be due to a blob moving in front of the probe. To each time a blob is detected corresponds one measured current value $I$ and one applied voltage value $V$ on each swept probe. The ensemble of current and voltage values gives an $I-V$ characteristic which can be fitted to estimate plasma parameters. Repeating this for different time lags around the time of the detection of the 
blob and for different radial locations of SLP gives then the 2D conditionally averaged evolution of ion saturation current, density, electron temperature, and floating potential associated with the blob.

In Fig. 3, one frame of the reconstructed 2D evolution of $\delta J_{\text {sat }}, n, V_{f}$, and $T_{e}$ for a $\mathrm{H}_{2}$ blob is shown. The reference signal is an $I_{\text {sat }}$ signal of a HEXTIP probe tip at $r=7 \mathrm{~cm}$. The measurement points of the SLP probe array are indicated by black crosses in Fig. 3(b). For $r \gtrsim 10 \mathrm{~cm}$, the SLP has to be tilted slightly in the toroidal direction so as not to hit the vessel wall. This is the reason for the nonuniformity of the measurement points in that region. In Figs. 3(a) and 3(b), we can clearly see a detached blob. Figure 3(c) shows the dipolar
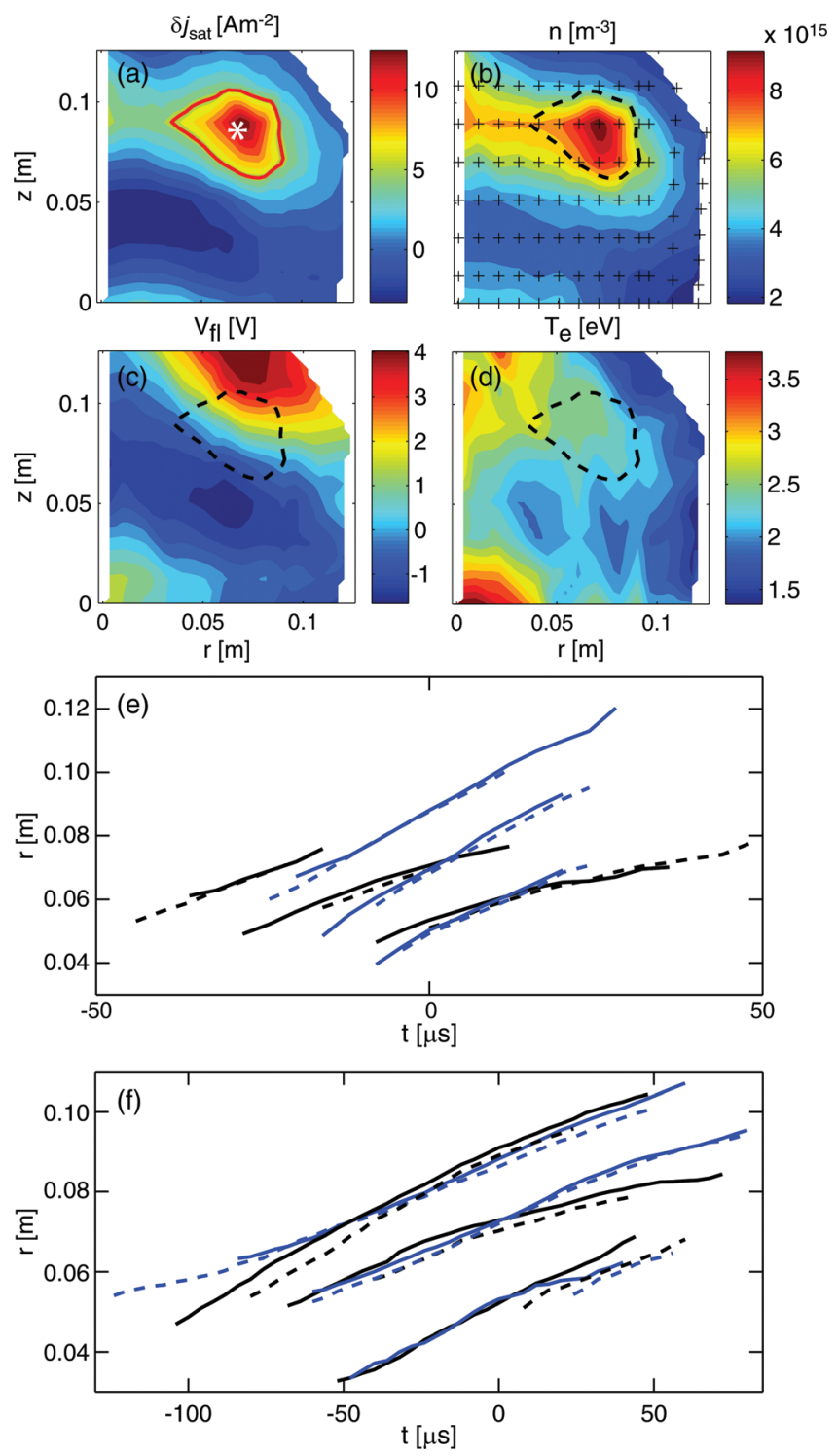

FIG. 3. (Color) (a)-(d) Example of the 2D profile of $\delta_{I \mathrm{~s} a t}, n, V_{f}$, and $T_{e}$ for a $\mathrm{H}_{2}$ blob obtained with the modified conditional sampling method (Ref. 43). (e) and (f) Radial blob position as a function of time determined from conditionally averaged blob propagation in $\mathrm{H}_{2}$ (e) and $\mathrm{Ar}$ (f). Blue lines correspond to shots with a single limiter and black lines to shots with two limiters. Reference probes at three different radial positions are used. Solid and dashed curves are obtained with a different threshold value to trace the blob. For two-limiter shots in $\mathrm{H}_{2}$, only data for $r<10 \mathrm{~cm}$ is available. This is the reason for the shorter trajectories in (e) compared to the one-limiter results. structure in $V_{f l}$ and Fig. 3(d) the perturbed temperature profile associated with the blob. To analyze the radial movement of the blob, analogous to the pattern recognition method, we identify the blob with a threshold condition on $\delta J_{\text {sat }}$, determine its center of mass, and follow it on a frame by frame basis. We require that the blob be detached from the main plasma and that its contour not intercept the boundary of our reconstruction domain. In Fig. 3(a), we have indicated the blob contour and its center identified in this way by a red contour and a white star. We then determine the blob center position as a function of time and evaluate an average radial blob velocity from a linear fit to this curve. Examples of such curves are shown in Figs. 3(e) and 3(f) for shots in $\mathrm{H}_{2}$ and Ar, for different connection lengths, and for analysis with different radial positions of the reference probe. To evaluate the blob temperature, we take the average temperature within the blob contour [see Fig. 3(d)]. For the evaluation of $a$ and $\delta n / n$, we use a pin of SLP as a reference probe to determine the conditionally averaged vertical $I_{\text {sat }}$ profile of the blob at a given radial position along the eight vertically aligned SLP probe tips. From this, we compute the half width at half maximum $a$ and $\delta n / n$ of the blob. We expect this technique to provide a more accurate evaluation of these parameters than if evaluated from the $2 \mathrm{D}$ profiles, such as the one shown in Fig. 3(a). In the latter case, the blob peak value is underestimated when it falls between the probe tips of SLP.

\section{VARIATION OF CONNECTION LENGTH}

In order to study the dependence of blob velocity upon the connection length, we installed a second limiter in TORPEX, identical to the first one and toroidally displaced by $180^{\circ}$. We compare blob motion with and without this second limiter. Along the magnetic field in the toroidal direction, we have HEXTIP at $83^{\circ}$ and SLP at $118^{\circ}$ from the second limiter.
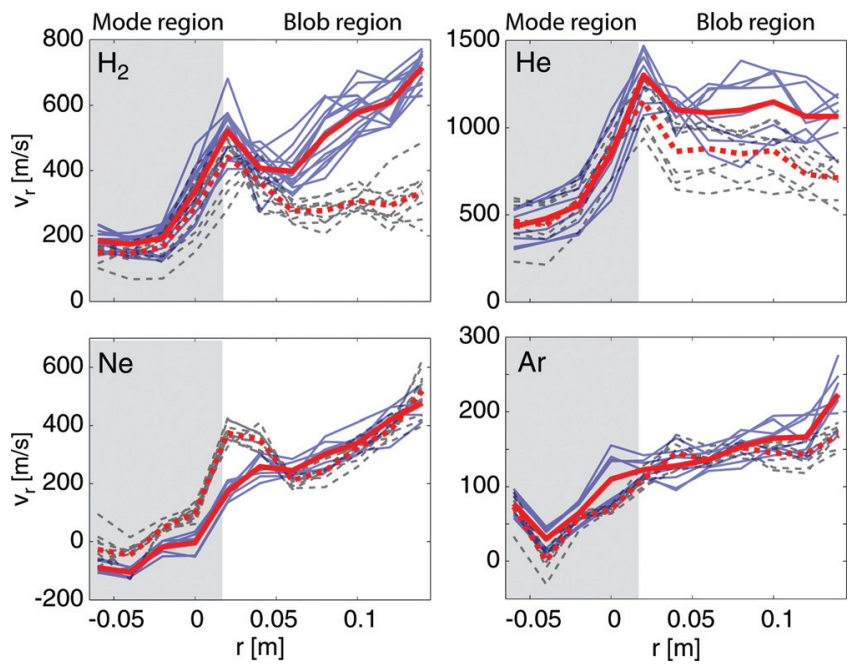

FIG. 4. (Color online) Radial profiles of average radial velocity of structures detected with pattern recognition in $\mathrm{H}_{2}, \mathrm{He}, \mathrm{Ne}$, and Ar. Solid blue lines correspond to discharges with a single limiter and black dashed lines to discharges with two limiters. Thick red lines indicate the average over the ensemble of curves. The mode region, where the pattern recognition method detects mainly modelike structures, is indicated by the shaded area. The region where blobs are detected lies at $r \gtrsim 2 \mathrm{~cm}$. 

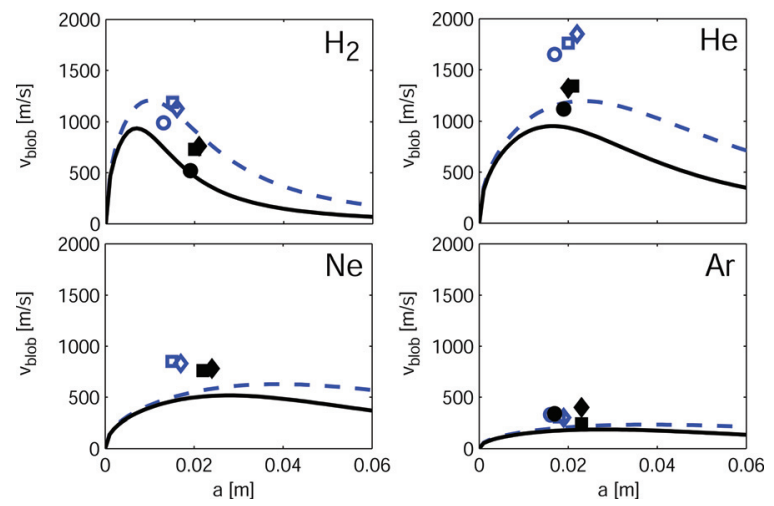

FIG. 5. (Color online) Measured blob velocity vs size for shots with one limiter (open symbols) and with two limiters (filled symbols). Equation (2) is overplotted for the measured blob parameters in the one limiter case (dashed) and the two limiter case (solid). Measured blob parameters are also tabulated in Appendix C.

In Fig. 4, we show the radial velocity of structures obtained with the pattern recognition method. Discharges from different experimental sessions and in between different machine openings were performed and included in the analysis. This gives the solid, blue and the dashed, black curves for the one- and the two-limiter cases, respectively. The thick red lines show the average over the ensemble of measurements. We find low values of radial velocity for $r \lesssim$ $2 \mathrm{~cm}$. This corresponds to the mode region where wavelike structures are detected. They move predominantly upwards. As we go further radially outwards, we detect more and more bloblike structures and the radial velocity increases. Here, we can see a clear difference between one- and twolimiter shots in $\mathrm{H}_{2}$. This difference gradually decreases as we go to higher ion masses. This result is in qualitative agreement with trends expected from the scaling law in Eq. (2), as discussed in Sec. III and in Fig. 2. Blobs in lighter gases are closer to the regime where parallel currents are the dominant current closure, and where blob velocity is proportional to $L$.

To perform a more quantitative comparison with the scaling law, we now apply the modified conditional sampling technique. We perform the analysis for blobs detected at three different radial positions on HEXTIP, $r=5.25,7$, and $8.75 \mathrm{~cm}$, respectively. The obtained blob parameters are tabulated in Appendix C. In Fig. 5, we plot the measured blob radial velocity versus size. Open symbols correspond to blobs from one-limiter shots and filled ones to blobs from two-limiter shots. Overplotted is the scaling law Eq. (2) (for $\left.\gamma_{\text {inst }}=\gamma_{\text {int }}\right)$, including the measured blob $\mathrm{T}_{\mathrm{e}}, \delta n / n$, and an estimation of the ion-neutral collision frequency, as discussed in Appendix C. These measurements confirm the trends observed in Fig. 4. The second limiter mainly affects blob velocities in $\mathrm{H}_{2}$ and $\mathrm{He}$, with little effect on blobs in $\mathrm{Ne}$ and Ar. From the tabulated blob parameters in Appendix C, we see that inserting the second limiter also reduces blob temperature, more importantly in $\mathrm{H}_{2}(\approx 20 \%$ reduction) than in the heavier gases $(\approx 10 \%$ reduction in Ar). The reduction of blob velocity and the trend across the different gases seem thus to result from both a reduction in blob temperature and an increased damping by parallel currents, which is more important for larger $\tilde{a}$. For example, in the hydrogen case and for $a=1.5 \mathrm{~cm}$, the scaling law in Eq. (2) predicts that $\approx 60 \%$ and $\approx 40 \%$ of the reduction of the blob velocity are due to the decrease of the connection length and the blob temperature, respectively. In absolute terms, Eq. (2) tends to underestimate blob velocity, but still provides a reasonably good estimate of it for the different plasmas.

\section{VARIATION OF GAS PRESSURE}

The possibility of influencing blob velocity by varying the neutral background pressure has already been demonstrated for seeded argon blobs in VTF (Versatile Toroidal Facility). ${ }^{20}$ Here, we explore this possibility of blob control in helium, using four different neutral gas pressures, $p_{n} \approx 0.021,0.042,0.064$, and $0.085 \mathrm{~Pa}$. We note here that increasing the gas pressure not only affects the blobs in our experiments, but also the mode properties. We observe a monotonic decrease in the mode frequency with increasing gas pressure from $\approx 14 \mathrm{kHz}$ down to $\approx 6 \mathrm{kHz}$. The wavelength of the interchange wave remaining unchanged, this corresponds to a decrease in the vertical phase velocity of the mode.

Figure 6(a) shows the radial velocity profiles from pattern recognition. All analyzed discharges are from the same experimental session. As anticipated, a clear decrease of blob radial velocity with gas pressure is found. This is confirmed by the conditional sampling method, as shown in the velocity-versus-size plot in Fig. 6(b) and the velocity-versusgas pressure plot in Fig. 6(c).

\section{OTHER ATTEMPTS OF BLOB CONTROL}

So far, we have presented two ways of blob control that confirm our present understanding of blob motion in TORPEX. In this section, we discuss two experiments undertaken in TORPEX to control blobs using methods that are not yet well understood.

The first experiment is motivated by the theoretical prediction that blob parallel currents and thus blob velocities can be changed by varying the angle between the magnetic field lines and the wall. ${ }^{45-47}$ For this purpose, a specially designed limiter has been constructed, which allows forming angles down to $10^{\circ}$ between magnetic field and limiter. ${ }^{24,48}$ The setup is shown in Fig. 7 with a photograph of the limiter (left) and a sketch of the limiter as seen from the top (right). More details and an estimate of the expected effect on the blob velocity can be found in Ref. 48 . Note that our configuration is not equivalent to the SOL of a diverted tokamak, where the blob velocity should be influenced by the angle between the normal to the divertor plate and the poloidal magnetic field. ${ }^{47}$ However, in both configurations the effect on blob velocity results from the closure of the electron diamagnetic current approaching the wall by parallel currents flowing from the wall. ${ }^{45}$ We have not observed a significant dependence of the blob velocity on the tilt angle of the limiter. To date, it is not clear whether these results contradict the theoretical predictions or if they are caused by effects specific to our setup, such as perturbations due to the edges 

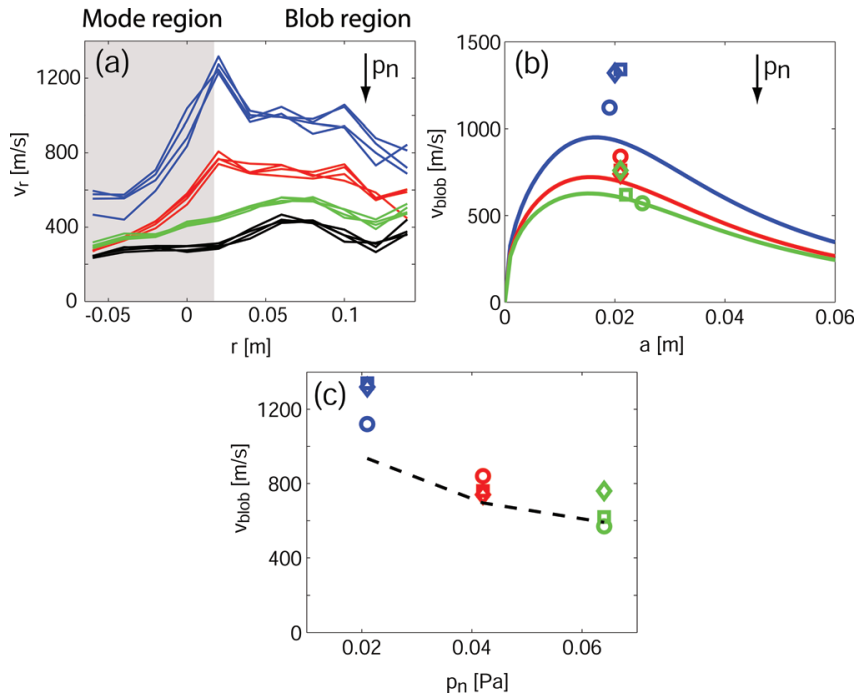

FIG. 6. (Color online) Results of a neutral gas pressure scan in helium. (a) Average radial velocity of structures from pattern recognition for four different gas pressures $p_{n}$. The gas pressure is varied from $\approx 0.021 \mathrm{~Pa}$ (uppermost, blue curves) to $\approx 0.085 \mathrm{~Pa}$ (lowest, black curves). In (b) and (c), we plot the result from the conditional sampling method, where data for three different gas pressures is available. The plotted curves show the predictions from Eq. (2).

of the plates (Fig. 7) or the fact that a blob needs to reconnect to the different plates as it moves radially.

In another experiment, a glass limiter is used instead of a steel limiter, originally intended to disconnect the blobs from the limiter and avoid parallel currents to damp blob velocity. However, the effect is that in the region where both ends of the field lines end on the limiter, the plasma potential is strongly reduced by the presence of the glass limiter. This is shown in Fig. 8, where we plot the plasma potential profile at a toroidal angle of $210^{\circ}$ from the limiter for discharges with the steel limiter (a) and with the glass limiter (b). In the glass limiter case, we even measure negative values of the plasma potential. The effect of this is that blobs are mainly convected around this region in the clockwise direction. This leads to a reduced radial cross-field particle transport in that region and, in particular, to a significant reduction of the ion saturation current, as shown in Figs. 8(c) and 8(d) for HEXTIP data.
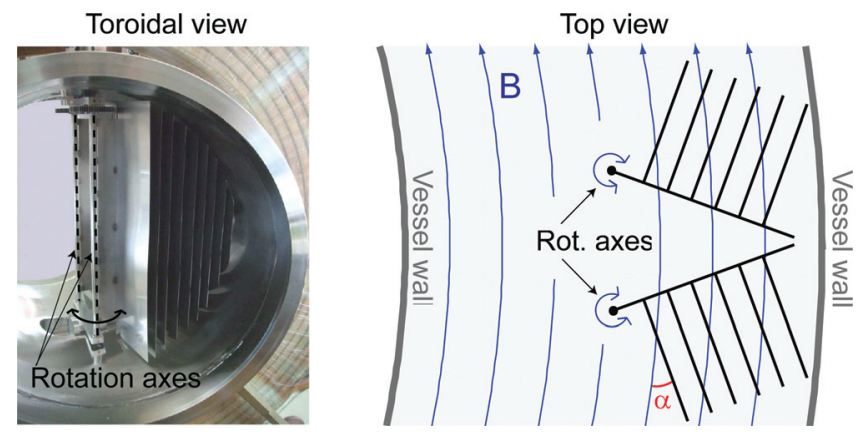

FIG. 7. (Color online) Left: Photograph of the limiter à configuration variable, installed on a mobile sector of the TORPEX vessel. The view is along the toroidal direction. Right: Sketch of the limiter (top view). Several stainless steel plates are mounted perpendicularly to two halfmoon limiters. The angle $\alpha$ between magnetic field lines and these plates can be varied by pivoting the two limiters around the vertical axes.
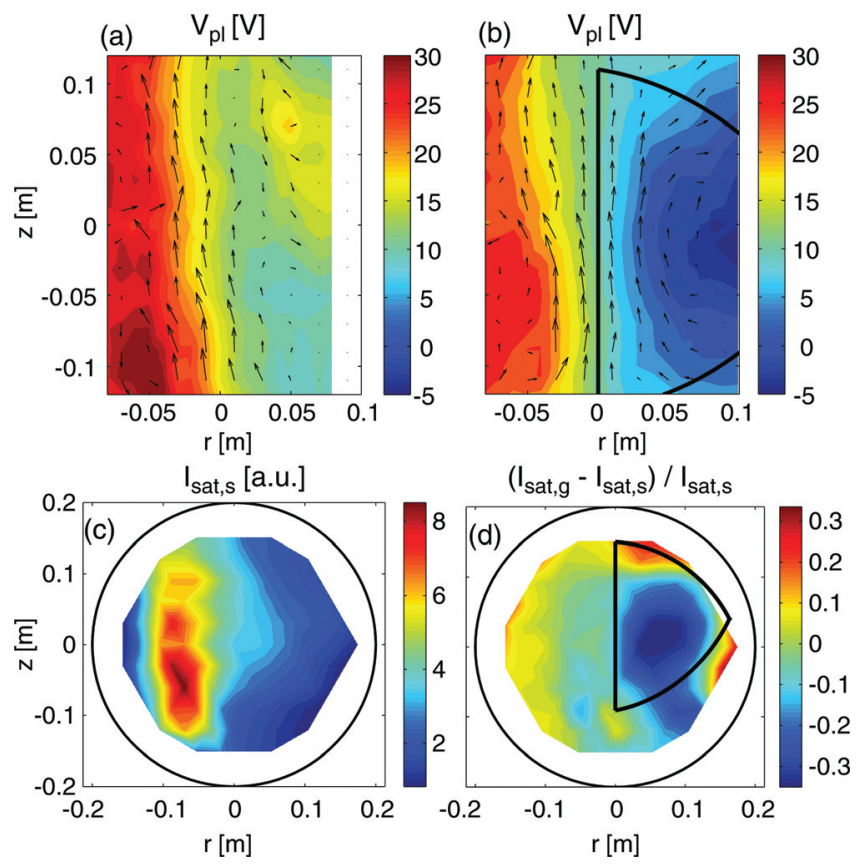

FIG. 8. (Color online) (a) Time-averaged plasma potential profile in helium for discharges with a steel limiter installed. Arrows indicate the direction of $\mathbf{E} \times \mathbf{B}$ flows. (b) The same as in (a), but for discharges with a glass limiter. (c) Profile of the ion saturation current in the steel limiter case. (d) Relative difference in the $I_{\text {sat }}$ profile between glass limiter and steel limiter discharges.

\section{SUMMARY AND OUTLOOK}

Based on a fairly complete understanding of the mechanisms regulating blob dynamics, we have investigated possibilities of controlling blob motion in TORPEX simple magnetized plasmas. The blob velocity formula derived and experimentally verified in our previous work ${ }^{21}$ predicts that a reduction of connection length should allow reducing blob velocity in helium and, more importantly, in hydrogen, while little effect is expected for the heavy gases Ne and Ar. This has been tested by introducing a second limiter in TORPEX in order to halve the connection length, and results indeed follow the predicted trends. The scaling law also predicts that an increase in neutral pressure reduces blob radial velocity. This has been experimentally confirmed for blobs in helium.

We have further explored blob control by changing the boundary conditions. A limiter was designed that allows changing the angle between the magnetic field and limiter, predicted to influence blob velocity in TORPEX. ${ }^{45,47,48}$ However, such an effect has not been observed so far.

In an attempt to electrically disconnect blobs from the limiter and increase their velocity, an insulating (glass) limiter was installed in TORPEX. It was found that this limiter charges up strongly negatively and produces vertical $\mathbf{E} \times \mathbf{B}$ flows that deviate the blobs around the region where field lines are on both ends connected to the glass limiter. Despite this negative result, these experiments show that a biasing of a part of the field lines is possible in TORPEX and that it can even be achieved passively with insulating surfaces.

As a way to influence blobs and SOL turbulence in tokamaks, it has been proposed to induce poloidal electric 
fields. ${ }^{49}$ This should create convective cells, increase the SOL width, and reduce heat loads on the divertor. Such ideas have already been tested in tokamaks (see, e.g., Refs. 50 and 51). We are currently investigating this in TORPEX with a dedicated setup. A 2D array of 24 electrodes is installed on the limiter. Each electrode can be biased individually and the current drawn from the plasma is acquired. This setup will enable us to address the question of the minimal perpendicular scale length of potential variations that can be achieved, and how far these variations propagate along the magnetic field.

In the derivation of the blob velocity scaling law, we have considered two different growth rates for secondary instabilities of the blob that can limit its velocity. In both cases, we found similar expressions for the velocity formula. Experimentally, the detailed shape of the blob and instabilities growing on it cannot be identified with the Langmuir probe measurements presented here. These limitations could soon be overcome due to fast framing camera imaging in TORPEX. ${ }^{52,53}$

\section{ACKNOWLEDGMENTS}

We acknowledge fruitful discussions with Kyle Gustafson and Joaquim Loizu. This work is partly supported by the Fonds National Suisse de la Recherche Scientifique.

\section{APPENDIX A: INERTIAL SCALING}

We want to present here a similar argument to that in Ref. 29 to support the scaling $v_{\text {blob }}=\sqrt{(2 a / R)} c_{s}$ in the absence of sheath currents and neutrals. For this, we consider Eq. (1) in the limit $L \rightarrow \infty$ and $v_{i n} \rightarrow 0$ and close the system with $(D / D t) n=0$. We normalize spacial scales to $a, a$ being the blob size, temporal scales to $\gamma_{\text {int }}^{-1}=\sqrt{R a} /\left(\sqrt{2} c_{s}\right)$, electrostatic potential to $\gamma_{\mathrm{int}} B a^{2}$, and density to $n_{0}$. We are left with

$$
\begin{aligned}
& \frac{\partial \tilde{n}}{\partial \tilde{z}}=\tilde{\nabla} \cdot\left(\tilde{n} \frac{D \tilde{\nabla_{\perp}} \tilde{\phi}}{D \tilde{t}}\right), \\
& \frac{D \tilde{n}}{D \tilde{t}}=0 .
\end{aligned}
$$

Here, the tilde symbol indicates dimensionless quantities. From the solution of Eqs. (A1), one can get the blob velocity $\tilde{v}_{\text {peak }}(\tilde{t})$, e.g., the velocity of the density peak. Going to physical units, one gets the family of solutions

$$
v_{\text {peak }}(t)=\sqrt{\frac{2 a}{R}} c_{s} \tilde{v}_{\text {peak }}\left(\sqrt{\frac{2}{R a}} c_{s} t\right) .
$$

Therefore, if $\tilde{v}_{\text {peak }}(\tilde{t})$ reaches a quasisteady phase, this velocity scales as $v_{\text {peak }} \propto \sqrt{2 a / R} c_{s}$.

\section{APPENDIX B: ALTERNATIVE SCALING}

In the formula for blob velocity in Eq. (2), we have assumed that a blob is subject to a secondary instability with growth rate $\gamma_{\text {inst }}$ that can limit its radial velocity, and we have set $\gamma_{\text {inst }}=\gamma_{\text {int }}$. Here, we consider the case in which a blob is subject to a Kelvin-Helmholtz instability and set $\gamma_{\text {inst }}=v_{\text {blob }} / a$. In this case Eq. (2) becomes quadratic in $v_{\text {blob }}$ and the positive solution is given by

$$
\begin{aligned}
v_{\text {blob }} & =-u+\sqrt{2 a \frac{c_{s}^{2}}{R} \frac{\delta n}{n}+u^{2},} \\
u & =\frac{a^{3} c_{s}}{2 \rho_{s}^{2} L}+\frac{a v_{\text {in }}}{2} .
\end{aligned}
$$

Comparing Eqs. (2) and (B1) in normalized units $\tilde{a}$, $\tilde{v}_{\text {blob}}$, and $\tilde{\eta}$ [see Eqs. (3) and (4) for definition], one finds that Eq. (B1) gives slightly higher blob velocities. However, Eqs. (2) and (B1) are very similar and, for $\delta n / n \geq 0.7$, blob velocities do not disagree by more than $36 \%$. It is worth noting that Eq. (B1) can also be obtained by calculating the linear growth rate of the interchange instability including sheath losses and ion-neutral collisions and applying the blob correspondence principle. ${ }^{30}$

\section{APPENDIX C: SUMMARY OF MEASURED BLOB PARAMETERS}

\begin{tabular}{|c|c|c|c|c|c|c|c|}
\hline Gas & $L(\mathbf{m})$ & $r(\mathbf{c m})$ & $p_{n}(\mathbf{P a})$ & $v(\mathrm{~m} / \mathrm{s})$ & $a(\mathrm{~cm})$ & $T_{e}(\mathrm{eV})$ & $\delta \boldsymbol{n} / \boldsymbol{n}$ \\
\hline $\mathrm{H}_{2}$ & $2 \pi$ & 5.25 & 0.014 & 990 & 1.3 & 2.6 & 0.79 \\
\hline $\mathrm{H}_{2}$ & $2 \pi$ & 7 & 0.014 & 1190 & 1.5 & 2.4 & 0.79 \\
\hline $\mathrm{H}_{2}$ & $2 \pi$ & 8.75 & 0.014 & 1130 & 1.6 & 2.5 & 0.75 \\
\hline $\mathrm{H}_{2}$ & $\pi$ & 5.25 & 0.013 & 520 & 1.9 & 2.0 & 0.80 \\
\hline $\mathrm{H}_{2}$ & $\pi$ & 7 & 0.013 & 730 & 2.0 & 1.9 & 0.81 \\
\hline $\mathrm{H}_{2}$ & $\pi$ & 8.75 & 0.013 & 760 & 2.1 & 2.1 & 0.79 \\
\hline $\mathrm{He}$ & $2 \pi$ & 5.25 & 0.019 & 1650 & 1.7 & 5.5 & 0.74 \\
\hline $\mathrm{He}$ & $2 \pi$ & 7 & 0.019 & 1760 & 2.0 & 5.2 & 0.74 \\
\hline $\mathrm{He}$ & $2 \pi$ & 8.75 & 0.019 & 1850 & 2.2 & 5.0 & 0.73 \\
\hline $\mathrm{He}$ & $\pi$ & 5.25 & 0.021 & 1120 & 1.9 & 4.6 & 0.76 \\
\hline $\mathrm{He}$ & $\pi$ & 7 & 0.021 & 1340 & 2.1 & 4.2 & 0.75 \\
\hline $\mathrm{He}$ & $\pi$ & 8.75 & 0.021 & 1320 & 2.0 & 4.3 & 0.75 \\
\hline $\mathrm{He}$ & $\pi$ & 5.25 & 0.042 & 840 & 2.1 & 4.1 & 0.73 \\
\hline $\mathrm{He}$ & $\pi$ & 7 & 0.042 & 760 & 2.1 & 3.6 & 0.73 \\
\hline $\mathrm{He}$ & $\pi$ & 8.75 & 0.042 & 740 & 2.0 & 3.7 & 0.74 \\
\hline $\mathrm{He}$ & $\pi$ & 5.25 & 0.064 & 570 & 2.5 & 3.8 & 0.77 \\
\hline $\mathrm{He}$ & $\pi$ & 7 & 0.064 & 620 & 2.2 & 3.5 & 0.75 \\
\hline $\mathrm{He}$ & $\pi$ & 8.75 & 0.064 & 760 & 2.1 & 3.6 & 0.73 \\
\hline $\mathrm{Ne}$ & $2 \pi$ & 7 & 0.014 & 850 & 1.5 & 3.8 & 0.81 \\
\hline $\mathrm{Ne}$ & $2 \pi$ & 8.75 & 0.014 & 830 & 1.7 & 3.6 & 0.80 \\
\hline $\mathrm{Ne}$ & $\pi$ & 7 & 0.013 & 760 & 2.2 & 3.2 & 0.84 \\
\hline $\mathrm{Ne}$ & $\pi$ & 8.75 & 0.013 & 780 & 2.4 & 3.2 & 0.82 \\
\hline $\mathrm{Ar}$ & $2 \pi$ & 5.25 & 0.014 & 330 & 1.6 & 1.9 & 0.70 \\
\hline $\mathrm{Ar}$ & $2 \pi$ & 7 & 0.014 & 310 & 1.8 & 1.7 & 0.65 \\
\hline $\mathrm{Ar}$ & $2 \pi$ & 8.75 & 0.014 & 300 & 1.9 & 1.7 & 0.65 \\
\hline $\mathrm{Ar}$ & $\pi$ & 5.25 & 0.015 & 340 & 1.7 & 1.7 & 0.67 \\
\hline $\mathrm{Ar}$ & $\pi$ & 7 & 0.015 & 240 & 2.3 & 1.5 & 0.65 \\
\hline $\mathrm{Ar}$ & $\pi$ & 8.75 & 0.015 & 400 & 2.3 & 1.6 & 0.60 \\
\hline
\end{tabular}

In Table I, we summarize blob parameters determined with the modified conditional sampling method. ${ }^{43}$ The different columns indicate the working gas, the connection

TABLE I. Blob parameters. 
length in the blob region, the radial position of the reference probe for conditional sampling, the neutral pressure, and blob velocity, size, temperature, and $\delta n / n$, i.e., the ratio of blob density above background and total blob density.

For the evaluation of Eq. (2), we further need an estimation of the ion-neutral collision frequency $v_{i n}$. This is obtained as follows. We assume a drifting Maxwellian for the ions with drift velocity $v_{\text {blob }}$, ion thermal velocity $v_{\text {th }, i}$, and neutral thermal velocity $v_{\mathrm{th}, n}$ satisfying $v_{\mathrm{th}, i} \gg v_{\mathrm{blob}}, v_{\mathrm{th}, n}$. We then find that $v_{\text {in }} \approx \frac{p_{n}}{T_{\mathrm{amb}}} \sigma^{\mathrm{mt}} v_{\mathrm{th}, i}$, with $T_{\mathrm{amb}}=0.025 \mathrm{eV}$ the ambient temperature and $\sigma^{\mathrm{mt}}$ the momentum transfer cross section in the center of mass system. For the curves in Figs. 5 and 6 , we have taken the values of $p_{n}$ in Table I, $\sigma^{\mathrm{mt}}$ $\approx 10^{-18} \mathrm{~m}^{2}$ (Refs. 54 and 55), and $T_{i}=1 \mathrm{eV}$.

${ }^{1}$ J. L. Terry, S. J. Zweben, K. Hallatschek, B. LaBombard, R. J. Maqueda, B. Bai, C. J. Boswell, M. Greenwald, D. Kopon, W. M. Nevis, C. S. Pitcher, B. N. Rogers, D. P. Stotler, and X. Q. Xu, Phys. Plasmas 10, 1739 (2003).

${ }^{2}$ J. A. Boedo, D. L. Rudakov, R. A. Moyer, G. R. McKee, R. J. Colchin, M. J. Schaffer, P. G. Stangeby, W. P. West, S. L. Allen, T. E. Evans, R. J. Fonck, E. M. Hollmann, S. Krasheninnikov, A. W. Leonard, W. Nevins, M. A. Mahdavi, G. D. Porter, G. R. Tynan, D. G. Whyte, and X. Xu, Phys. Plasmas 10, 1670 (2003).

${ }^{3}$ S. J. Zweben, R. J. Maqueda, D. P. Stotler, A. Keesee, J. Boedo, C. E. Bush, S. M. Kaye, B. LeBlanc, J. L. Lowrance, V. J. Mastrocola, R. Maingi, N. Nishino, G. Renda, D. W. Swain, J. B. Wilgen, and the NSTX Team, Nucl. Fusion 44, 134 (2004).

${ }^{4}$ A. Kirk, H. R. Wilson, G. F. Counsell, R. Akers, E. Arends, S. C. Cowley, J. Dowling, B. Lloyd, M. Price, and M. Walsh, Phys. Rev. Lett. 92, 245002 (2004).

${ }^{5}$ R. Sánchez, B. P. van Milligen, D. E. Newman, and B. A. Carreras, Phys. Rev. Lett. 90, 185005 (2003).

${ }^{6}$ M. Spolaore, V. Antoni, E. Spada, H. Bergsåker, R. Cavazzana, J. R. Drake, E. Martines, G. Regnoli, G. Serianni, and N. Vianello, Phys. Rev. Lett. 93, 215003 (2004).

${ }^{7}$ I. Furno, B. Labit, M. Podestà, A. Fasoli, S. H. Müller, F. M. Poli, P. Ricci, C. Theiler, S. Brunner, A. Diallo, and J. Graves, Phys. Rev. Lett. 100, 055004 (2008).

${ }^{8}$ G. Y. Antar, S. I. Krasheninnikov, P. Devynck, R. P. Doerner, E. M. Hollmann, J. A. Boedo, S. C. Luckhardt, and R. W. Conn, Phys. Rev. Lett. 87, 065001 (2001).

${ }^{9}$ T. A. Carter, Phys. Plasmas 13, 010701 (2006).

${ }^{10}$ A. Loarte, B. Lipschultz, A. S. Kukushkin, G. F. Matthews, P. C. Stangeby, N. Asakura, G. F. Counsell, G. Federici, A. Kallenbach, K. Krieger, A. Mahdavi, V. Philipps, D. Reiter, J. Roth, J. Strachan, D. Whyte, R. Doerner, T. Eich, W. Fundamenski, A. Herrmann, M. Fenstermacher, P. Ghendrih, M. Groth, A. Kirschner, S. Konoshima, B. LaBombard, P. Lang, A. W. Leonard, P. Monier-Garbet, R. Neu, H. Pacher, B. Pegourie, R. A. Pitts, S. Takamura, J. Terry, E. Tsitrone, and the ITPA Scrape-off Layer and Divertor Physics Topical Group, Nucl. Fusion 47, S203 (2007).

${ }^{11}$ S. J. Zweben, J. A. Boedo, O. Grulke, C. Hidalgo, B. LaBombard, R. J. Maqueda, P. Scarin, and J. L. Terry, Plasma Phys. Controlled Fusion 49, S1 (2007).

${ }^{12}$ J. A. Boedo, J. Nucl. Mater. 390-391, 29 (2009).

${ }^{13}$ S. I. Krasheninnikov, Phys. Lett. A 283, 368 (2001).

${ }^{14}$ S. I. Krasheninnikov, D. A. D'Ippolito, and J. R. Myra, J. Plasma Phys. 74, 679 (2008)

${ }^{15}$ W. Fundamenski, W. Sailer, and the JET EFDA contributors, Plasma Phys. Controlled Fusion 46, 233 (2004).

${ }^{16}$ J. R. Myra, D. A. D'Ippolito, D. P. Stotler, S. J. Zweben, B. P. LeBlanc, J. E. Menard, R. J. Maqueda, and J. Boedo, Phys. Plasmas 13, 092509 (2006).

${ }^{17}$ O. Grulke, J. L. Terry, B. LaBombard, and S. J. Zweben, Phys. Plasmas 13, 012306 (2006).

${ }^{18}$ A. Kirk, B. Koch, R. Scannell, H. R. Wilson, G. Counsell, J. Dowling, A. Herrmann, R. Martin, and M. Walsh, Phys. Rev. Lett. 96, 185001 (2006).
${ }^{19}$ A. Schmid, A. Herrmann, H. W. Müller, and the ASDEX Upgrade Team, Plasma Phys. Controlled Fusion 50, 045007 (2008).

${ }^{20}$ N. Katz, J. Egedal, W. Fox, A. Le, and M. Porkolab, Phys. Rev. Lett. 101, 015003 (2008).

${ }^{21}$ C. Theiler, I. Furno, P. Ricci, A. Fasoli, B. Labit, S. H. Müller, and G. Plyushchev, Phys. Rev. Lett. 103, 065001 (2009).

${ }^{22}$ R. J. Maqueda, D. P. Stotler, and the NSTX Team, Nucl. Fusion 50, 075002 (2010).

${ }^{23}$ A. Fasoli, B. Labit, M. McGrath, S. H. Müller, G. Plyushchev, M. Podestà, and F. M. Poli, Phys. Plasmas 13, 055902 (2006).

${ }^{24}$ A. Fasoli, A. Burckel, L. Federspiel, I. Furno, K. Gustafson, D. Iraji, B. Labit, J. Loizu, G. Plyushchev, P. Ricci, C. Theiler, A. Diallo, S. H. Mueller, M. Podestà, and F. Poli, Plasma Phys. Controlled. Fusion 52, 124020 (2010).

${ }^{25}$ D. A. D'Ippolito, J. R. Myra, and S. I. Krasheninnikov, Phys. Plasmas 9, 222 (2002).

${ }^{26}$ G. Q. Yu and S. I. Krasheninnikov, Phys. Plasmas 10, 4413 (2003).

${ }^{27}$ A. Y. Aydemir, Phys. Plasmas 12, 062503 (2005).

${ }^{28}$ S. Sugita, M. Yagi, S.-I. Itoh, and K. Itoh, Plasma Fusion Res. 3, 040 (2008).

${ }^{29}$ O. E. Garcia, N. H. Bian, V. Naulin, A. H. Nielsen, and J. J. Rasmussen, Phys. Plasmas 12, 090701 (2005).

${ }^{30}$ J. R. Myra and D. A. D’Ippolito, Phys. Plasmas 12, 092511 (2005).

${ }^{31}$ M. Podestà, A. Fasoli, B. Labit, M. McGrath, S. H. Müller, and F. M. Poli, Plasma Phys. Controlled Fusion 47, 1989 (2005).

${ }^{32}$ D. A. D’Ippolito and J. R. Myra, Phys. Plasmas 10, 4029 (2003).

${ }^{33}$ P. Ricci and B. N. Rogers, Phys. Rev. Lett. 104, 145001 (2010).

${ }^{34}$ F. M. Poli, P. Ricci, A. Fasoli, and M. Podestà, Phys. Plasmas 15, 032104 (2008).

${ }^{35}$ B. Labit, I. Furno, A. Fasoli, A. Diallo, S. H. Müller, G. Plyushchev, M. Podestà, and F. M. Poli, Phys. Rev. Lett. 98, 255002 (2007).

${ }^{36}$ M. Podestà, A. Fasoli, B. Labit, I. Furno, P. Ricci, F. M. Poli, A. Diallo, S. H. Müller, and C. Theiler, Phys. Rev. Lett. 101, 045001 (2008).

${ }^{37}$ S. H. Müller, A. Diallo, A. Fasoli, I. Furno, B. Labit, and M. Podestà, Phys. Plasmas 14, 110704 (2007).

${ }^{38}$ C. Theiler, A. Diallo, A. Fasoli, I. Furno, B. Labit, M. Podestà, F. M. Poli, and P. Ricci, Phys. Plasmas 15, 042303 (2008).

${ }^{39}$ S. H. Müller, A. Diallo, A. Fasoli, I. Furno, B. Labit, G. Plyushchev, M. Podestà, and F. M. Poli, Phys. Plasmas 13, 100701 (2006).

${ }^{40}$ L. Federspiel, B. Labit, P. Ricci, A. Fasoli, I. Furno, and C. Theiler, Phys. Plasmas 16, 092501 (2009).

${ }^{41}$ S. H. Müller, A. Fasoli, B. Labit, M. McGrath, O. Pisaturo, G. Plyushchev, M. Podestà, and F. M. Poli, Phys. Plasmas 12, 090906 (2005).

${ }^{42}$ H. Johnsen, H. L. Pécseli, and J. Trulsen, Phys. Fluids 30, 2239 (1987).

${ }^{43}$ I. Furno, B. Labit, A. Fasoli, F. M. Poli, P. Ricci, C. Theiler, S. Brunner, A. Diallo, J. P. Graves, M. Podestà, and S. H. Müller, Phys. Plasmas 15, 055903 (2008).

${ }^{44}$ C. Theiler, I. Furno, A. Kuenlin, P. Marmillod, and A. Fasoli, Rev. Sci. Instrum. 82, 013504 (2011).

${ }^{45}$ R. H. Cohen and D. D. Ryutov, Phys. Plasmas 2, 2011 (1995).

${ }^{46}$ D. Farina, R. Pozzoli, and D. Ryutov, Plasma Phys. Controlled. Fusion 35, 1271 (1993).

${ }^{47}$ R. H. Cohen and D. D. Ryutov, Contrib. Plasma Phys. 46, 678 (2006).

${ }^{48}$ C. Theiler, I. Furno, P. Ricci, A. Fasoli, and B. Labit, in 36th EPS Conference on Plasma Phys. Sofia, 2009 [Euriphys Conf. Abstr. 33E, 0-5.067 (2009)].

${ }^{49}$ R. H. Cohen and D. D. Ryutov, Nucl. Fusion 37, 621 (1997).

${ }^{50}$ G. F. Counsell, J.-W. Ahn, R. Akers, E. Arends, S. J. Fielding, P. Helander, A. Kirk, H. Meyer, A. Tabasso, H. Wilson, and Y. Yang, J. Nucl. Mater. 313-316, 804 (2003).

${ }^{51}$ S. J. Zweben, R. J. Maqueda, A. L. Roquemore, C. E. Bush, R. Kaita, R. J. Marsala, Y. Raitses, R. H. Cohen, and D. D. Ryutov, Plasma Phys. Controlled. Fusion 51, 105012 (2009).

${ }^{52}$ D. Iraji, A. Diallo, A. Fasoli, I. Furno, and S. Shibaev, Rev. Sci. Instrum. 79, 10F508 (2008).

${ }^{53}$ D. Iraji, I. Furno, A. Fasoli, and C. Theiler, Phys. Plasmas 17, 122304 (2010).

${ }^{54}$ A. E. Glassgold, P. S. Krstić, and D. R. Schultz, Astrophys. J. 621, 808 (2005).

${ }^{55}$ A. V. Phelps, J. Appl. Phys. 76, 747 (1994). 